\title{
Synthesis and Spectroscopic Studies of Highly Fluorescent, Solvatochromic Diastereomers with Differentially Stacked Bithiophene-Substituted Quinoxaline Rings
}

\author{
Ryan A. Ciufo, John J. Kreinbihl, Sarah R. Johnson, and Jocelyn M. Nadeau* \\ Department of Chemistry, Biochemistry, and Physics, Marist College, 3399 North Road, \\ Poughkeepsie, NY 12601, United States
}

*Corresponding Author. Tel.: +1-845-575-3000; fax: +1-845-575-3184; e-mail address: jocelyn.nadeau@marist.edu

\begin{abstract}
Diastereomeric C-shaped molecules containing closely stacked bithiophene-substituted quinoxaline rings were synthesized and characterized by NMR, UV-vis absorption, and fluorescence spectroscopy. The unique geometry of each diastereomer resulted in different degrees of $\pi$-overlap between the bithiophene-substituted quinoxaline ring chromophores, modulating their spectroscopic properties. The donor-acceptor nature of this chromophore gave rise to its positive solvatochromism. ${ }^{1} \mathrm{H}$ NMR and UV-vis absorption spectroscopy confirmed the existence of $\pi-\pi$ interactions in the ground state between the quinoxaline rings in both molecules but between the bithiophene rings only in the syn isomer. They exhibited significant emission maxima bathochromic shifts, a strong, positive solvatochromism, increased band broadening, and larger Stokes shifts when compared to a compound with an unstacked chromophore. Additionally, the syn isomer consistently showed $\lambda_{\text {max,em }}$ value red-shifts and larger band broadening and Stokes shifts compared to the anti isomer due to the greater $\pi$-overlap in the syn isomer.
\end{abstract}




\section{Keywords}

Quinoxaline; Bithiophene; Pi overlap; Intramolecular excimer; Charge transfer; Solvatochromic

\section{Introduction}

It is well established that organic molecules, especially those with $\pi$-conjugation, display significant structure-property relationships. ${ }^{1-4}$ A better understanding of these relationships is achieved by studying molecules with unique topologies whose molecular properties can be manipulated by synthetic methods. Aromatic molecules are particularly versatile in this regard because they can be synthetically tuned to produce different properties via direct through-bond conjugation $^{5-10}$ or through-space interactions between closely stacked aromatic rings. ${ }^{11-20}$ Probing the effects of interchromophore interactions between stacked aromatic rings has been the subject of numerous research studies, owing to their importance in a variety of areas of chemistry, most recently as a key design element in molecular electronics applications. ${ }^{21-28}$

We recently reported the synthesis and characterization of diastereomeric C-shaped molecules containing closely, but differently, stacked thiophene-substituted quinoxaline rings (Figure 1; anti- and syn-BT) $^{12}$ that exhibited interesting spectroscopic properties arising, in part, from their unique molecular architecture. Additionally, the donor-acceptor nature of the thiophene-quinoxaline chromophore resulted in their solvatochromism, significantly expanding their emission color range. The different degrees of $\pi$-overlap between the chromophores of the diastereomers, where the quinoxaline rings are overlapped in both anti- and syn-BT, but the thiophene rings are overlapped only in syn-BT, led to their different electronic structures and, in turn, different spectroscopic properties. ${ }^{19,24,29,30}$ These results highlight the importance of having 
well-defined structures in organic electronics applications, as opposed to having a mixture of different molecular orientations in these complex systems, which can have a significant impact on overall device performance. ${ }^{24,27,31-34}$ Moreover, using solvent polarity as a means of controlling chromophore emission properties is an important design element because it is easier to change the solvation environment than the structure of a molecule or polymer. ${ }^{6,35}$ In this work, we present the results for the next generation of these diastereomeric C-shaped molecules, which contain differentially stacked bithiophene-substituted quinoxaline rings (Figure 1; anti- and synBBT).<smiles></smiles>

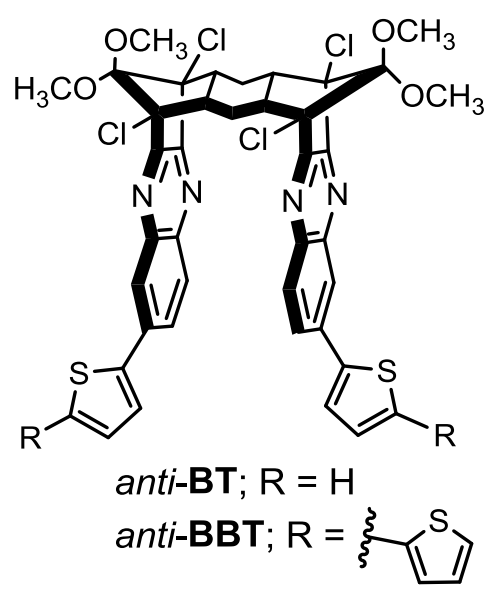<smiles>BrC(Br)(Br)c1ccc(-c2ccc(-c3cccs3)s2)cc1</smiles>

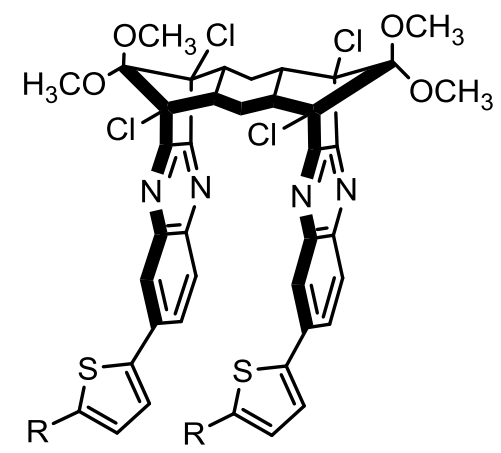

syn-BT; $\mathrm{R}=\mathrm{H}$

syn-BBT; $\mathrm{R}=\xi \mathrm{S}$

Figure 1. C-shaped molecules anti- and syn-BT and anti- and syn-BBT; model compounds CQT and CQBT.

Extending each chromophore by an additional thiophene ring was carried out to increase $\pi$ conjugation both through-bond and through-space to observe the subsequent impact on their photophysical properties, particularly on their emission spectra. ${ }^{16}$ Model compounds CQT and CQBT were synthesized and studied as references for understanding the photophysical 
properties of the lone chromophores in the absence of $\pi$-overlap. Herein we present the synthesis and ${ }^{1} \mathrm{H}$ NMR spectroscopy characterization of CQT, CQBT, anti-BBT, and syn-BBT and the results of our analyses of their UV-vis absorption and fluorescence properties as a function of structure and solvent polarity.

\section{Results and discussion}

\subsection{Synthesis and NMR spectroscopy}

Diastereomers anti- and syn-BBT were synthesized from their previously reported bromine-substituted precursors (Scheme 1). ${ }^{12}$ A microwave-assisted, Pd-catalyzed Suzuki coupling reaction was used to prepare anti- and syn-BBT from anti- and syn-BBr, respectively, and 2,2'-bithiophene-5-boronic acid. Anti- and syn-BBT were purified by chromatography on silica gel, and their isolated yields were $24 \%$ and $19 \%$, respectively.
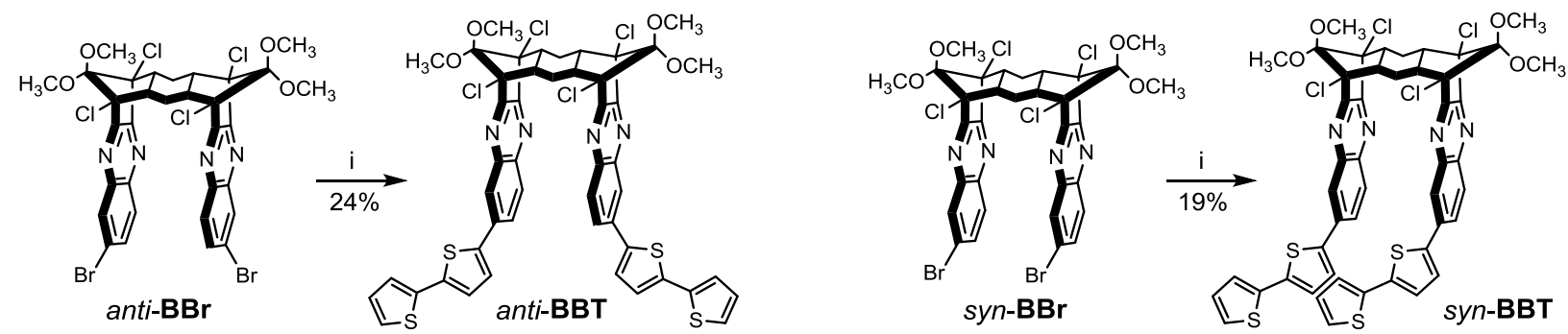

Scheme 1. Synthesis of anti- and syn-BBT. i) 2,2'-bithiophene-5-boronic acid, $\mathrm{Pd}\left(\mathrm{PPh}_{3}\right)_{4}$, $\mathrm{Na}_{2} \mathrm{CO}_{3}, \mathrm{EtOH}$, toluene, MW $120{ }^{\circ} \mathrm{C}, 30 \mathrm{~min}$.

Model compounds CQT and CQBT were obtained following similar Pd-catalyzed Suzuki coupling procedures with their bromine-substituted precursor, CQB (Scheme 2). CQB was synthesized by a Zn-catalyzed condensation reaction under microwave-assisted conditions that was previously reported for the synthesis of anti- and $s y n-\mathbf{B B r} .^{12}$ 


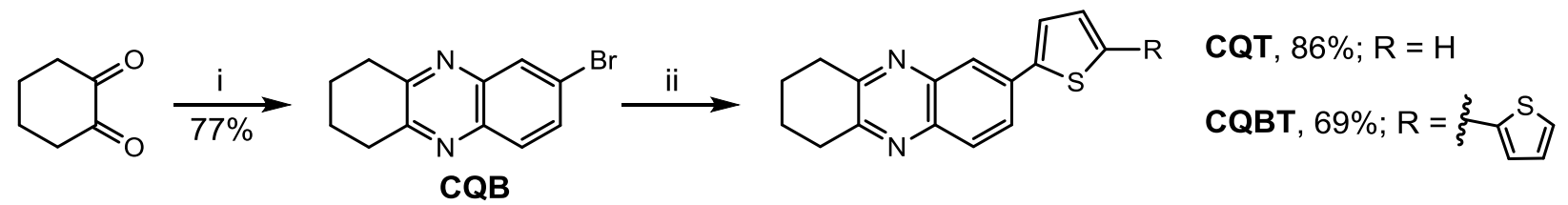

Scheme 2. Synthesis of model compounds CQT and CQBT. i) 1,2-diamino-4-bromobenzene, $\mathrm{Zn}(\mathrm{OAc})_{2}$, chlorobenzene, $\mathrm{MW} 200{ }^{\circ} \mathrm{C}, 30 \mathrm{~min}$; ii) 2-thienylboronic acid (for CQT) or 2,2'bithiophene-5-boronic acid (for CQBT), $\mathrm{Pd}\left(\mathrm{PPh}_{3}\right)_{4}, \mathrm{Na}_{2} \mathrm{CO}_{3}, \mathrm{EtOH}$, toluene, MW $120{ }^{\circ} \mathrm{C}, 30$ min.

1,2-Cyclohexanedione was reacted with 1,2-diamino-4-bromobenzene in the presence of a catalytic amount of $\mathrm{Zn}(\mathrm{OAc})_{2}$ to give $\mathbf{C Q B}$ in a $77 \%$ yield after chromatography on silica gel.

CQB was subjected to a microwave-assisted, Pd-catalyzed Suzuki coupling reaction with 2thienylboronic acid to give CQT in $86 \%$ yield and with 2,2'-bithiophene-5-boronic acid to give CQBT in 69\% yield after purification by chromatographic methods. All compounds reported herein were characterized by ${ }^{1} \mathrm{H}$ and ${ }^{13} \mathrm{C}$ NMR spectroscopy and HRMS analysis, with the data fully corroborating the proposed structures.

The NMR spectroscopy data obtained for anti- and syn-BBT were consistent with our previously reported results for anti- and $s y n-\mathbf{B T},{ }^{12}$ except for the anticipated differences arising from the new thiophene rings. The syn isomers have a $\sigma$ plane of symmetry that cuts in between the aromatic rings through the methylene carbons of the cyclohexane bridging unit, whereas the anti isomers have a $\mathrm{C}_{2}$ axis of symmetry. As observed for syn-BT, ${ }^{13} \mathrm{C}$ NMR spectroscopy experiments on syn-BBT illustrate just how similar, yet still chemically different, the two methylene carbons of the central cyclohexane ring are. At $50 \mathrm{MHz}$, these two carbons appeared as one unresolved peak at $19.9 \mathrm{ppm}$, but at $100 \mathrm{MHz}$, there were clearly two peaks at 19.92 and 19.87 ppm (Figure S-5, Supplementary data). The cyclohexane methylene carbons are furthest 
from the point of difference in these molecules, where the thiophene ring attaches to the quinoxaline ring, so they are in chemical environments that are only slightly different. In antiBBT, the two cyclohexane methylene carbons are chemically equivalent, resulting in only one peak at $19.9 \mathrm{ppm}$ in its $100 \mathrm{MHz}{ }^{13} \mathrm{C}$ NMR spectrum, as expected (Figure S-4, Supplementary data). Interestingly, the cyclohexane carbons in the model compounds were also difficult to distinguish at $50 \mathrm{MHz}$. CQB, CQT, and CQBT all lack symmetry, so their carbon atoms are all in different chemical environments. The four cyclohexane carbons should give rise to four aliphatic chemical shifts, specifically two differently shielded pairs where the carbons within each pair are in very similar chemical environments. In the $50 \mathrm{MHz}{ }^{13} \mathrm{C}$ NMR spectrum of $\mathbf{C Q B}$, there were two unresolved aliphatic chemical shifts at 33.4 and $22.9 \mathrm{ppm}$. In the $50 \mathrm{MHz}{ }^{13} \mathrm{C}$ NMR spectra of CQT and CQBT, these same aliphatic carbons were just barely distinguishable at $33.45,33.39,23.02$, and $23.00 \mathrm{ppm}$ for CQT (Figure S-2, Supplementary data) and at 33.46, $33.40,23.02$, and $23.00 \mathrm{ppm}$ for CQBT, where the peaks at $23 \mathrm{ppm}$ were just barely separated as a split at the top of the peak. The $100 \mathrm{MHz}{ }^{13} \mathrm{C}$ NMR spectrum of CQBT showed two clearly resolved peaks at 33.5 and $33.4 \mathrm{ppm}$ and two nearly resolved peaks at 23.00 and $22.98 \mathrm{ppm}$ (Figure S-3, Supplementary data). As observed for the syn isomers of the C-shaped molecules, the chemically nonequivalent cyclohexane carbons at $23 \mathrm{ppm}$ in CQB, CQT, and CQBT were difficult to distinguish because they are furthest from the asymmetric point of attachment of the substituent to the quinoxaline ring.

The aromatic proton chemical shifts in CQBT, anti-BBT, and syn-BBT were examined to assess the shielding effects and confirm the presence of $\pi$ - $\pi$ interactions in the ground state. These interactions were observed in anti- and syn-B $\mathbf{T}^{12}$ and reported for other molecules with stacked aromatic rings. ${ }^{15,16,33}$ Table 1 lists the chemical shift assignments for the aromatic 
protons of CQBT, which represent the shielding environment of these protons in the absence of $\pi$ overlap, compared to the corresponding proton assignments for anti- and syn-BBT. Protons on the quinoxaline and bithiophene rings of anti- and syn-BBT exhibited the expected upfield shifts to varying degrees due to the shielding effects of the aromatic rings that are directly across from each other, which mirrored the results obtained for anti- and syn-BT. ${ }^{12}$ For example, the quinoxaline protons $\left(\mathrm{H}_{\mathrm{a}}, \mathrm{H}_{\mathrm{b}}\right.$, and $\left.\mathrm{H}_{\mathrm{c}}\right)$ in anti- and syn-BBT showed upfield shifts between 0.2-0.4 ppm compared to CQBT, with $\mathrm{H}_{\mathrm{c}}$ in anti-BBT being shifted upfield by the largest amount compared to all of the protons $(0.44 \mathrm{ppm})$. The thiophene ring protons showed variable degrees of upfield shifts in anti- versus syn-BBT compared to CQBT, which was attributed to the bithiophene rings being overlapped in syn-BBT but not in anti-BBT. Accordingly, $\mathrm{H}_{\mathrm{d}}-\mathrm{H}_{\mathrm{h}}$ in synBBT showed consistent upfield shifts compared to the corresponding protons in CQBT, especially $\mathrm{H}_{\mathrm{g}}$ and $\mathrm{H}_{\mathrm{h}}(0.32$ and $0.37 \mathrm{ppm}$, respectively). In anti-BBT, the only thiophene ring proton to show an upfield shift compared to $\mathbf{C Q B T}$ was $\mathrm{H}_{\mathrm{g}}(0.15 \mathrm{ppm})$.

Table 1. Chemical shifts of aromatic protons.

\begin{tabular}{cccc}
\hline${ }^{1} \mathbf{H}$ & \multicolumn{3}{c}{ Chemical Shift (ppm) } \\
Type & CQBT & anti-BBT & syn-BBT \\
\hline $\mathrm{H}_{\mathrm{a}}$ & 8.15 & 7.92 & 7.93 \\
$\mathrm{H}_{\mathrm{b}}$ & 7.94 & 7.70 & $7.68-7.74^{a}$ \\
$\mathrm{H}_{\mathrm{c}}$ & 7.90 & 7.46 & $7.68-7.74^{a}$ \\
$\mathrm{H}_{\mathrm{d}}$ & $7.22-7.25^{b}$ & 7.27 & 7.05 \\
$\mathrm{H}_{\mathrm{e}}$ & 7.04 & 7.09 & 6.96 \\
$\mathrm{H}_{\mathrm{f}}$ & $7.22-7.25^{b}$ & 7.31 & 7.19 \\
$\mathrm{H}_{\mathrm{g}}$ & 7.39 & 7.24 & 7.07 \\
$\mathrm{H}_{\mathrm{h}}$ & 7.18 & 7.20 & 6.81
\end{tabular}

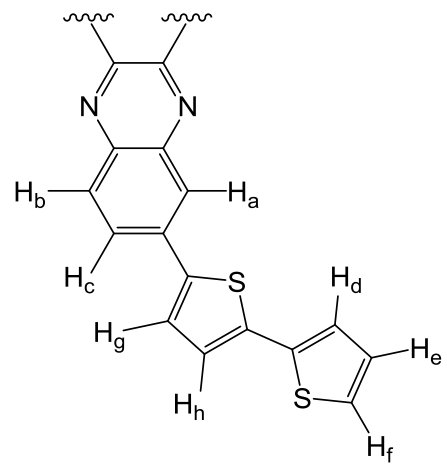

${ }^{a}$ Multiplet is assigned to both $\mathrm{H}_{\mathrm{b}}$ and $\mathrm{H}_{\mathrm{c}} \cdot{ }^{b}$ Multiplet is assigned to both $\mathrm{H}_{\mathrm{d}}$ and $\mathrm{H}_{\mathrm{f}}$.

These results clearly demonstrate that there are significant $\pi-\pi$ interactions in the ground state in anti- and syn-BBT between the aromatic rings that are directly across from one another. The data 
are easily rationalized upon viewing these structures directly through the aromatic rings (Figure 2). For the purpose of comparison, the ground-state geometries of anti- and syn-BBT were optimized using density functional theory (DFT) at the B3LYP/6-31G* level of theory. ${ }^{36}$ The quinoxaline rings overlap to roughly the same degree in both anti-and syn-BBT, which explains the similar upfield shifts observed for $\mathrm{H}_{\mathrm{a}}$ and $\mathrm{H}_{\mathrm{b}}$. The upfield shift observed for $\mathrm{H}_{\mathrm{c}}$ was more pronounced for anti-BBT (0.44 ppm) compared to syn-BBT (0.16-0.22 ppm), which is consistent with $\mathrm{H}_{\mathrm{c}}$ sitting in the shielding zone of the opposite thiophene ring in anti-BBT but not in synBBT. Accordingly, the only thiophene proton in anti-BBT that showed an upfield shift compared to CQBT was $\mathrm{H}_{\mathrm{g}}$, which is the only thiophene proton in the shielding zone of the opposite thiophene ring. In syn-BBT, all the thiophene protons showed upfield shifts compared to the corresponding protons in CQBT. Collectively, these results confirm ground-state $\pi$ overlap between the quinoxaline rings in both $\mathrm{C}$-shaped molecules and that the bithiophene rings are overlapped in syn-BBT but not in anti-BBT.
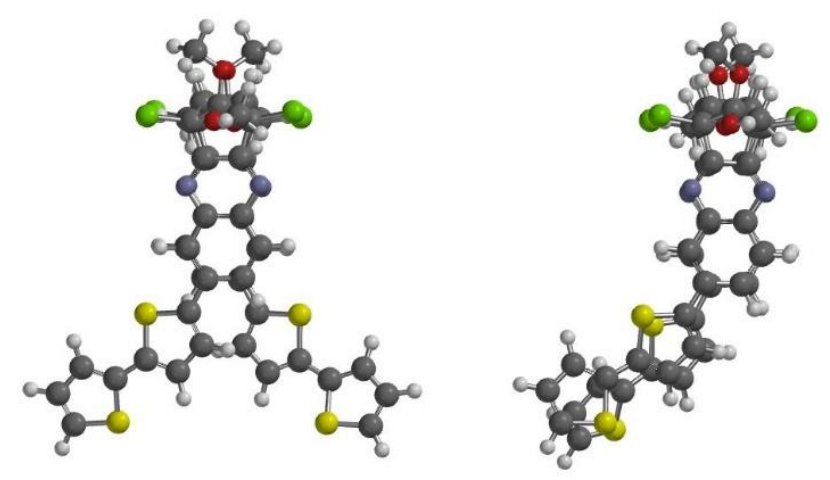

Figure 2. DFT-optimized (B3LYP/6-31G*) ground-state geometries of anti-BBT (left) and synBBT (right).

\subsection{UV-vis absorption and fluorescence spectroscopy}


Steady-state UV-vis absorption and fluorescence spectroscopy studies were performed on CQT, CQBT, anti-BBT, and syn-BBT in a variety of solvents of increasing polarity from hexanes to methanol (Table 2; Figures 3 and 4). These experiments were performed to study the impact of the structural differences between these compounds, namely their varying degrees of through-bond and through-space $\pi$-delocalization, and solvent polarity on their absorption and emission properties, ${ }^{37}$ as compared to those observed for anti- and syn-BT. ${ }^{12}$

Table 2 lists the $\lambda_{\max , a b s}$ values for each compound in all the solvents studied, and Figure 3 shows their absorption spectra in representative solvents ranging from nonpolar to polar (hexanes/dichloromethane/methanol). The effect of increasing the through-bond conjugation in going from a thiophene- to a bithiophene-substituted quinoxaline ring can be seen by comparing the absorption spectra of CQT to those of CQBT (Table 2 and Figure 3). CQT consistently showed two $\lambda_{\max , a b s}$ bands that appeared in the ranges of $276-281$ and $353-365 \mathrm{~nm}$. CQBT showed two similar bands, but they look very different and fell within the ranges of 256-259 and 379-391 nm with other vibrational bands in between. CQBT also showed a third shorter wavelength $\lambda_{\text {max,abs }}$ band between 206-211 nm. Within each solvent, the long-wavelength $\lambda_{\text {max,abs }}$ band observed for CQBT was red-shifted by approximately $30 \mathrm{~nm}$ compared to the corresponding band for CQT, which is consistent with increased $\pi$-conjugation and delocalization in CQBT due to the additional thiophene ring. ${ }^{10,38}$ The onset of absorption of CQBT was significantly red-shifted $(>30 \mathrm{~nm})$ compared to CQT, which also confirms the increased $\pi$-conjugation (Figure 3 ). Moreover, comparing the long-wavelength $\lambda_{\max , \text { abs }}$ band observed for anti- and syn-BBT to the corresponding band observed for anti- and syn-BT ${ }^{12}$ also revealed a $30 \mathrm{~nm}$ bathochromic shift for the BBT derivatives, as expected. 
Table 2. Photophysical properties of CQT, CQBT, anti-BBT, and syn-BBT in solvents of different polarity.

\begin{tabular}{|c|c|c|c|c|c|c|}
\hline & solvent & $\lambda_{\max , \mathrm{abs}}(\mathrm{nm})$ & $\begin{array}{l}\lambda_{\text {max,em }} \\
(\mathrm{nm})^{a}\end{array}$ & $\begin{array}{l}\text { fwhm } \\
(\mathrm{nm})^{b}\end{array}$ & $\mathrm{SS}\left(\mathrm{cm}^{-1}\right)^{c}$ & $\Phi_{\mathrm{F}}^{d}$ \\
\hline \multirow[t]{11}{*}{ CQT } & hexanes & 276,353 & 391 & 54 & 2753 & 0.0035 \\
\hline & cyclohexane & 277,354 & 390 & 54 & 2608 & - \\
\hline & toluene & $357^{e}$ & 410 & 57 & 3621 & 0.024 \\
\hline & ethyl acetate & 277,354 & 416 & 60 & 4210 & 0.023 \\
\hline & chloroform & 281,362 & 428 & 63 & 4260 & 0.13 \\
\hline & dichloromethane & 280,358 & 425 & 64 & 4404 & 0.081 \\
\hline & acetone & $354^{e}$ & 430 & 69 & 4993 & 0.052 \\
\hline & acetonitrile & 277,353 & 431 & 69 & 5127 & 0.083 \\
\hline & n-butanol & 280,364 & 454 & 73 & 5446 & 0.53 \\
\hline & ethanol & 280,365 & 462 & 76 & 5752 & 0.49 \\
\hline & methanol & 279,363 & 467 & 82 & 6135 & 0.56 \\
\hline \multirow[t]{11}{*}{ CQBT } & hexanes & $210,256,381$ & 417,440 & 49 & - & 0.22 \\
\hline & cyclohexane & $256,383^{e}$ & 419,444 & 51 & - & - \\
\hline & toluene & $386^{e}$ & 451 & 65 & 3734 & 0.27 \\
\hline & ethyl acetate & $257,381^{e}$ & 463 & 73 & 4648 & 0.12 \\
\hline & chloroform & $259,390^{e}$ & 488 & 82 & 5149 & 0.14 \\
\hline & dichloromethane & $258,385^{e}$ & 485 & 86 & 5355 & 0.15 \\
\hline & acetone & $381^{e}$ & 501 & 97 & 6287 & 0.16 \\
\hline & acetonitrile & $211,257,379$ & 500 & 91 & 6385 & 0.18 \\
\hline & n-butanol & $206,259,390$ & 529 & 102 & 6672 & 0.25 \\
\hline & ethanol & $208,259,391$ & 537 & 111 & 6953 & 0.082 \\
\hline & methanol & $208,259,388$ & 547 & 120 & 7492 & 0.021 \\
\hline \multirow{11}{*}{$\begin{array}{l}\text { anti- } \\
\text { BBT }\end{array}$} & hexanes & 259,389 & 434,457 & 62 & - & 0.22 \\
\hline & cyclohexane & 256,383 & 436,461 & 62 & - & - \\
\hline & toluene & $396^{e}$ & 470 & 73 & 3976 & 0.13 \\
\hline & ethyl acetate & 345,390 & 499 & 81 & 5601 & 0.16 \\
\hline & chloroform & 349,401 & 505 & 81 & 5136 & 0.22 \\
\hline & dichloromethane & 349,399 & 517 & 86 & 5720 & 0.35 \\
\hline & acetone & 348,392 & 520 & 94 & 6279 & 0.27 \\
\hline & acetonitrile & 347,397 & 545 & 103 & 6840 & 0.37 \\
\hline & n-butanol & 348,398 & 550 & 105 & 6944 & 0.21 \\
\hline & ethanol & 347,398 & 566 & 113 & 7458 & 0.044 \\
\hline & methanol & 347,398 & 574 & 130 & 7704 & 0.016 \\
\hline \multirow{4}{*}{$\begin{array}{l}\text { syn- } \\
\text { BBT }\end{array}$} & hexanes & 260,390 & 463 & 87 & 4043 & 0.058 \\
\hline & cyclohexane & 261,390 & 468 & 84 & 4274 & - \\
\hline & toluene & $397^{e}$ & 499 & 87 & 5149 & 0.12 \\
\hline & ethyl acetate & 342,390 & 512 & 88 & 6110 & 0.15 \\
\hline
\end{tabular}




\begin{tabular}{lccccc} 
chloroform & 346,402 & 526 & 89 & 5864 & 0.23 \\
dichloromethane & 347,400 & 537 & 95 & 6378 & 0.26 \\
acetone & 345,392 & 534 & 98 & 6784 & 0.21 \\
acetonitrile & 342,397 & 556 & 106 & 7203 & 0.22 \\
$n$-butanol & 345,400 & 569 & 110 & 7425 & 0.14 \\
ethanol & 344,398 & 579 & 121 & 7854 & 0.044 \\
methanol & 342,399 & 587 & 147 & 8027 & 0.0087 \\
\hline nm. & ${ }^{\text {fwhm }}=$ full width at half maximum. ${ }^{c}$ Stokes shift, $\mathrm{SS}=1 / \lambda_{\text {max,abs }}-1 / \lambda_{\text {max,em. }}$
\end{tabular}

${ }^{a} \lambda_{\mathrm{ex}}=350 \mathrm{~nm} .{ }^{b} \mathrm{fwhm}=$ full width at half maximum. ${ }^{c}$ Stokes shift, $\mathrm{SS}=1 / \lambda_{\max , \mathrm{abs}}-1 / \lambda_{\max , \mathrm{em}}$. ${ }^{d}$ Fluorescence quantum yields were determined relative to coumarin 153 in methanol $\left(\Phi_{\mathrm{F}}=0.45\right)$ as the standard $\left(\lambda_{\mathrm{ex}}=350 \mathrm{~nm}\right) .{ }^{39}$ Solvent absorption obscured the short-wavelength analyte absorption. Note: The photographs depict quartz cuvettes containing a solution of each compound in dichloromethane that was being irradiated at $365 \mathrm{~nm}$ by a hand-held UV lamp.

Comparing the UV-vis absorption spectra of CQBT, which contains a single, unstacked chromophore, to those of anti- and syn-BBT in any given solvent allows one to analyze the $\pi$ stacking effects and their subsequent impact on the electronic properties of the C-shaped molecules (Table 2 and Figure 3). First, the onset of absorption for anti- and syn-BBT was consistently red-shifted compared those of CQBT by $10-20 \mathrm{~nm}$. This result supports the ${ }^{1} \mathrm{H}$ NMR evidence suggesting the presence of $\pi-\pi$ interactions in the ground state between the aromatic rings in anti- and syn-BBT that are absent in CQBT (vide supra). ${ }^{14,15,30}$

(a)

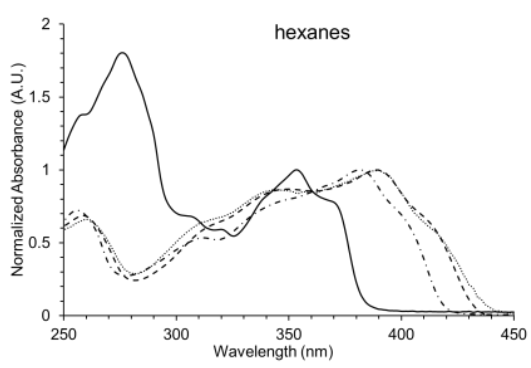

(b)

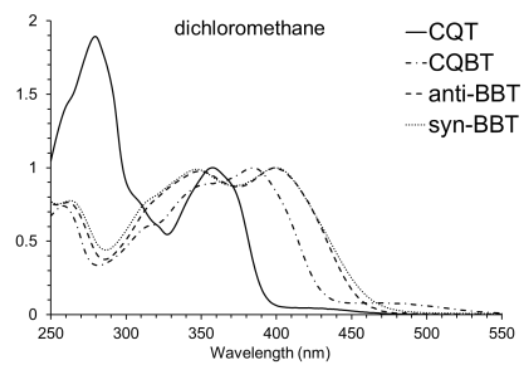

(c)

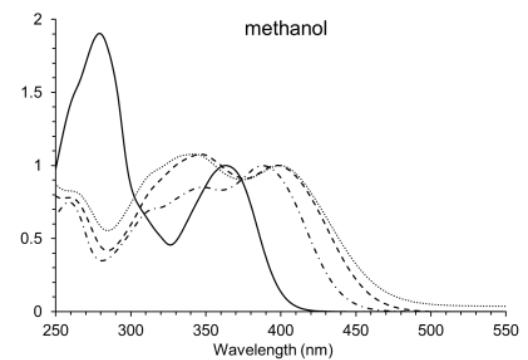


Figure 3. UV-vis spectra of CQT (solid line), CQBT (dash-dotted line), anti-BBT (dashed line), and syn-BBT (dotted line) in (a) hexanes, (b) dichloromethane, and (c) methanol. The spectra were normalized to the long-wavelength $\lambda_{\max , a b s}$ value.

Although the absorption spectra and $\lambda_{\max , a b s}$ values for CQBT do not appear to change significantly as a function of solvent polarity, a dramatic change in the absorption spectra can be observed upon examining the data for anti- and syn-BBT from hexanes to methanol (Table 2; Figure 3). In nonpolar solvents (from hexanes to toluene), the UV-vis spectra of CQBT, antiBBT, and syn-BBT were quite similar in overall profile, except for the earlier onset of absorption of the C-shaped molecules that was noted above (for example, see Figure 3a). As seen in Table 2 for hexanes and cyclohexane, anti- and syn-BBT showed two $\lambda_{\text {max,abs }}$ bands (antiBBT: 256-259 and 383-389 nm; syn-BBT: 260-261 and $390 \mathrm{~nm}$ ) that were similar to those of CQBT in all the solvents (256-259 and 379-391 nm). However, in the more polar solvents, the absorption spectra of anti- and syn-BBT changed to a very different profile compared to those of CQBT, which did not vary significantly from hexanes to methanol. Specifically, in the polar solvents (from ethyl acetate to methanol), the absorption spectra of anti- and syn-BBT broadened overall and a new blue-shifted $\lambda_{\text {max,abs }}$ band appeared at approximately $340 \mathrm{~nm}$ that was about equal intensity to or higher than the existing long-wavelength band at approximately $390 \mathrm{~nm}$ (anti-BBT: 345-349 and 390-401 nm; syn-BBT: 342-347 and 390-402 nm). These results suggest that in nonpolar solvents the electronic transitions available to anti- and syn-BBT are the same as in CQBT, namely those of a single bithiophene-substituted quinoxaline chromophore. However, as solvent polarity increases, additional transitions become available to the $\pi$-stacked aromatic rings in anti- and syn-BBT. ${ }^{16}$ The observed broadening and splitting of the absorption band has been invoked as evidence of electronically interacting chromophores in the ground 
state. $^{18,29-31}$ These findings were consistent with the emission spectroscopy results described below.

The fluorescence emission spectra of CQT, CQBT, anti-BBT, and syn-BBT all showed a broad, structureless, emission band in every solvent studied, except for CQBT and anti-BBT in hexanes and cyclohexane (Table 2 and Figure 4). Aside from the exceptions noted above that will be discussed shortly, CQBT, anti-BBT, and syn-BBT demonstrated significant emission maxima bathochromic shifts, a strong, positive solvatochromism, band broadening, and increasing Stokes shifts as solvent polarity increased from hexanes to methanol. To observe the effects of adding the thiophene ring on the emission spectra of the BBT family compared to the BT family, ${ }^{12}$ the difference between the $\lambda_{\text {max,em values of CQT and CQBT were evaluated in }}$ toluene and in methanol. In toluene, there was a $41 \mathrm{~nm}$ difference and in methanol, there was an $80 \mathrm{~nm}$ difference. The same comparison was made between anti-BT and anti-BBT because their thiophene rings do not overlap, so the differences between their $\lambda_{\text {max,em }}$ values should also reflect the increased $\pi$-conjugation. Accordingly, the difference between their $\lambda_{\text {max,em }}$ values in toluene was $43 \mathrm{~nm}$ and in methanol was $83 \mathrm{~nm}$, which match the comparison between CQT and CQBT. The emission spectra of CQT were narrower compared to those of CQBT in nearly all the solvents studied, where the full-width at half-maximum (fwhm) values observed in toluene to methanol $^{40}$ ranged between 57-82 $\mathrm{nm}$ for CQT and between 65-120 nm for CQBT, respectively. The same trend was observed for the Stokes shifts, which ranged between 3,621 to $6,135 \mathrm{~cm}^{-1}$ for CQT and 3,734 to 7,492 $\mathrm{cm}^{-1}$ for CQBT. Collectively, these results illustrate the increased through-bond $\pi$-conjugation and delocalization in the bithiophene compared to the thiophene analogs, as expected. ${ }^{10,38}$ They also suggest that CQBT has significantly more excited-state charge-transfer character given its more pronounced solvatochromism and the very large fwhm 
and Stokes shift values compared to those of CQT. ${ }^{37}$ Both the thiophene- and bithiophenesubstituted quinoxaline rings exhibit donor-accepter characteristics because thiophene and bithiophene are good electron donors and quinoxaline is a good electron acceptor. ${ }^{41,42}$ However, given that bithiophene is a better donor than thiophene, the intramolecular charge-transfer (ICT) excited-state character of CQBT is expected to be greater than that of CQT, as observed.

(a) CQT

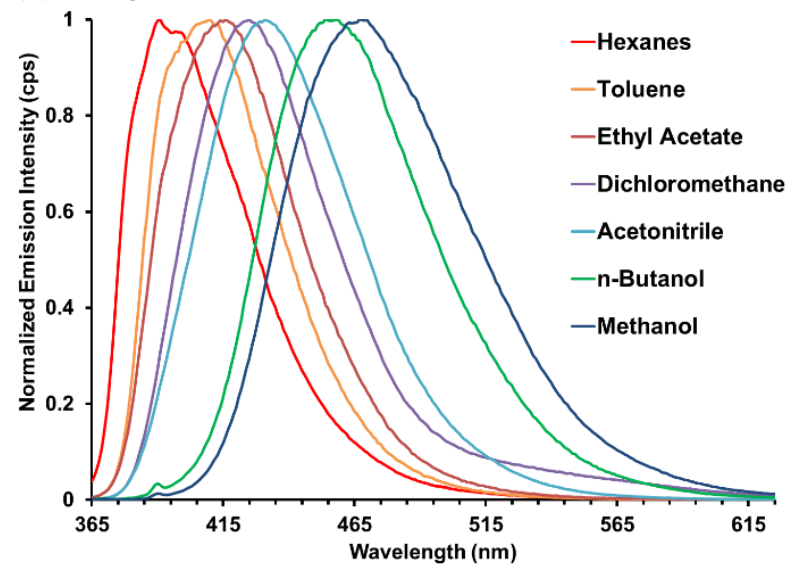

(c) anti-BBT

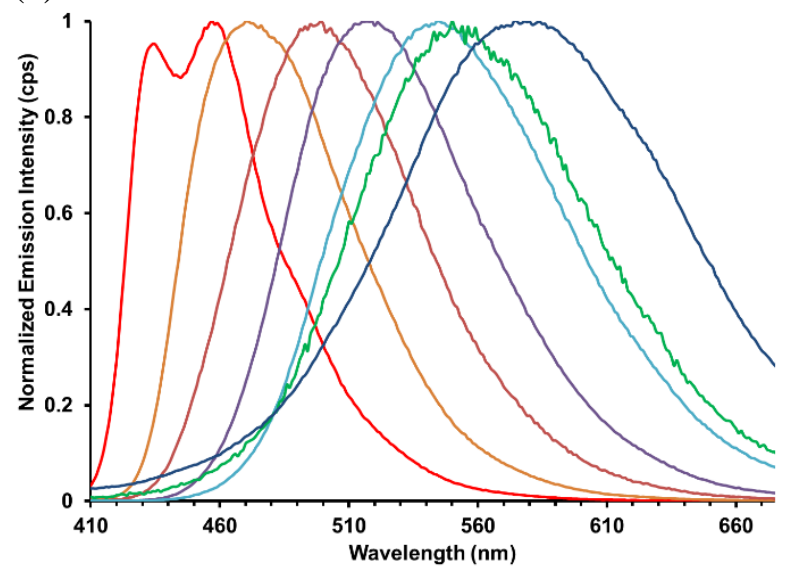

(b) CQBT

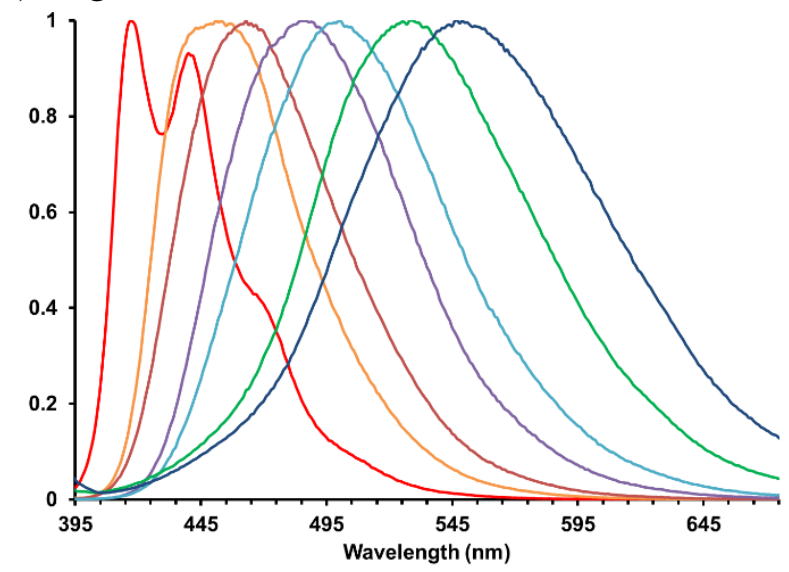

(d) syn-BBT

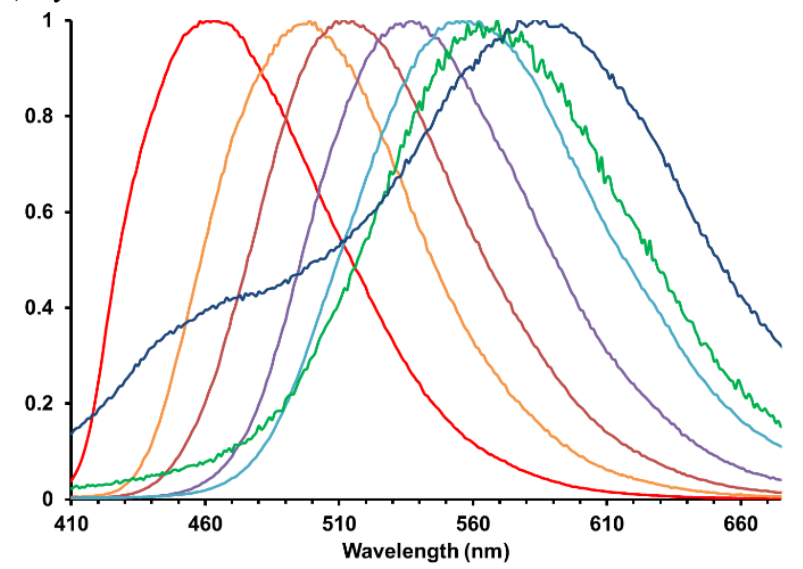

Figure 4. Fluorescence emission spectra of a) CQT, b) CQBT, c) anti-BBT, d) syn-BBT in various solvents $\left(\lambda_{\mathrm{ex}}=350 \mathrm{~nm}\right)$. For the sake of clarity, only spectra from representative solvents in the polarity range from Table 2 are presented. The emission intensities are normalized at $\lambda_{\text {max }, \mathrm{em}}$. 
The $\lambda_{\text {max,em }}$ values of CQBT, anti-BBT, and syn-BBT were examined in solvents ranging in polarity from toluene to methanol to study the effect of the different degrees of $\pi$-stacking in the C-shaped molecules. ${ }^{40}$ For CQBT, the $\lambda_{\text {max,em }}$ values were blue-shifted from those of antiBBT by an average of $27 \mathrm{~nm}$ and from those of syn-BBT by an average of $44 \mathrm{~nm}$. Additionally, the $\lambda_{\text {max,em }}$ values of $s y n$-BBT were red-shifted by an average of $17 \mathrm{~nm}$ compared to those of anti-BBT. Similar trends were observed within the BT family. ${ }^{12}$ The dramatic red-shifts observed for the C-shaped molecules compared to the unstacked bithiophene-substituted quinoxaline model, CQBT, can be attributed to the overlap between the aromatic rings in antiand $s y n$-BBT that gives rise to strong, stabilizing $\pi-\pi$ interactions in their excited states. ${ }^{28,30,31}$ Analogous results have been observed in numerous other molecules that were specifically designed to have closely stacked aromatic rings, where emission in these molecules was said to be from an intramolecular excimer state..$^{13,14,18,24,29,30,32}$ It is possible to deconstruct the effects that the different degrees of $\pi$-stacking in these molecules have on the emission maxima. The red-shift in the $\lambda_{\text {max,em }}$ values for anti-BBT compared to CQBT represents the through-space conjugation of the overlapping quinoxaline rings in anti-BBT that is absent in CQBT. The redshift in the $\lambda_{\max , \mathrm{em}}$ values for syn-BBT compared to anti-BBT corresponds to the effect of the $\pi$ stacked bithiophene rings in syn-BBT that is absent in anti-BBT. Finally, the red-shift in the $\lambda_{\text {max,em }}$ values for syn-BBT compared to CQBT signifies the effect of the overlapped bithiophene-substituted quinoxaline rings in syn-BBT that is absent in CQBT. Accordingly, the effect of the bithiophene ring overlap $\left(17 \mathrm{~nm} ; 613 \mathrm{~cm}^{-1}\right)$ and the quinoxaline ring overlap (27 $\mathrm{nm} ; 1,037 \mathrm{~cm}^{-1}$ ) numerically adds up to the overall effect of completely overlapping the bithiophene-substituted quinoxaline rings $\left(44 \mathrm{~nm} ; 1,650 \mathrm{~cm}^{-1}\right)$. Thus, by comparing and contrasting the $\lambda_{\text {max,em }}$ values of CQBT, anti-BBT, and syn-BBT, it was possible to deconstruct 
the effects that the different degrees of $\pi$-stacking have on their emission maxima. Similar trends were observed for the fwhm and Stokes shift values, which further supports the analysis above.

The emission spectra of CQBT, anti-BBT, and syn-BBT showed a dramatic dependence on solvent polarity in nearly all the solvents studied (Table 2 and Figure 4). In hexanes and cyclohexane, the emission spectra of CQBT and anti-BBT exhibited similar, highly structured spectra that both showed two $\lambda_{\text {max,em }}$ bands centered around 418 and $442 \mathrm{~nm}$ for CQBT and 435 and $459 \mathrm{~nm}$ for anti-BBT, in addition to a long-wavelength shoulder. In contrast, the emission spectra of syn-BBT in hexanes and cyclohexane were broad and structureless with $\lambda_{\text {max,em }}$ bands at 463 and $468 \mathrm{~nm}$, respectively. In solvents of increasing polarity from toluene to methanol, the emission spectra of CQBT, anti-BBT, and syn-BBT were all broad and structureless, and they exhibited a strong, positive solvatochromism. Together, these results suggest that in nonpolar solvents like hexanes and cyclohexane, CQBT and anti-BBT are likely to be emitting from the locally excited (LE) state of the bithiophene-quinoxaline chromophore. However, in the more polar solvents, the ICT excited-state character of this chromophore is stabilized, and therefore emission in polar solvents is likely to be from an excited state with significant charge-transfer (CT) character. ${ }^{6,37,43}$ The fact that all the emission bands of syn-BBT were broad, structureless, and solvatochromic in all of the solvents studied suggests that the increased through-space $\pi$ conjugation from the bithiophene ring overlap, which is absent in CQBT and anti-BBT, stabilizes the excited state, even in nonpolar solvents like hexanes and cyclohexane. ${ }^{28,30,31}$

Furthermore, the fwhm and Stokes shift values for CQBT, anti-BBT, and syn-BBT were analyzed as a function of solvent polarity, and the results were consistent with the $\lambda_{\max , \mathrm{em}}$ analysis above. For example, the fwhm values in toluene for CQBT, anti-BBT, and syn-BBT were 65,73 , and $87 \mathrm{~nm}$, respectively, and the fwhm values in methanol were 120, 130, and 147 
$\mathrm{nm}$, respectively. The Stokes shifts also increased as a function of increasing solvent polarity for all three compounds, but the increase was more dramatic for anti-BBT (toluene to methanol: 3,976 to $\left.7,704 \mathrm{~cm}^{-1}\right)$ and $s y n$-BBT $\left(5,149\right.$ to $\left.8,027 \mathrm{~cm}^{-1}\right)$ compared to CQBT $\left(3,734\right.$ to $7,492 \mathrm{~cm}^{-}$

${ }^{1}$ ) in every solvent, and the increase was greater for syn-BBT compared to anti-BBT. These results further corroborate the findings described above, namely, that the bithiophene-substituted quinoxaline ring shows significant CT character in the excited state, which is stabilized by increasing solvent polarity and greater $\pi$-overlap. ${ }^{6,43}$ The $\pi$-overlap between the chromophores in anti- and syn-BBT stabilizes the charge delocalization in the excited state, which leads to broader bands and larger Stokes shifts for the C-shaped molecules compared to CQBT. ${ }^{28,30,31}$ Moreover, the greater band broadening and larger Stokes shifts observed for syn-BBT compared to anti-BBT illustrates the stabilizing effect that the overlapping bithiophene rings have on the excited state of syn-BBT.

The relative quantum yields of CQT, CQBT, anti-BBT, and syn-BBT measured in a variety of solvents were found to be solvent-dependent, but they did not vary directly with solvent polarity (Table 2). ${ }^{44}$ For CQBT, anti-BBT, and syn-BBT, the quantum yields increased and then decreased with increasing solvent polarity. From toluene to $n$-butanol, the quantum yields for the BBT family of compounds fell within a range of 0.12-0.37, but in ethanol and methanol, the values ranged between $0.01-0.08$. The marked decrease in quantum yield in the polar protic solvents is likely to be due to the emergence of a new, non-radiative deactivation pathway in these hydrogen bond donating solvents. ${ }^{6,43}$

\section{Conclusion}


In this work, we reported on a new pair of highly fluorescent C-shaped molecules containing differentially stacked bithiophene-substituted quinoxaline rings, anti- and syn-BBT, and related model compounds, CQT and CQBT, that were synthesized and characterized by NMR, UV-vis absorption, and fluorescence spectroscopy. A detailed analysis of the aromatic ${ }^{1} \mathrm{H}$ NMR chemical shift shielding effects and the UV-vis absorption spectra revealed $\pi-\pi$ interactions in the ground state between the quinoxaline rings in anti- and syn-BBT, but $\pi-\pi$ interactions between the bithiophene rings only in syn-BBT. The different degrees of $\pi$-stacking in these molecules was also evident from their emission spectra, where anti- and syn-BBT consistently had red-shifted $\lambda_{\max , \mathrm{em}}$ values and larger band broadening and Stokes shifts compared to CQBT, and syn-BBT consistently showed red-shifted $\lambda_{\max , \mathrm{em}}$ values, increased band broadening and Stokes shifts compared to anti-BBT. The C-shaped molecules also showed a remarkably strong, positive solvatochromism that was also observed for CQBT, confirming the excited-state ICT character of the bithiophene-substituted quinoxaline ring. Moreover, the increased band broadening and Stokes shifts observed for anti- and syn-BBT compared to CQBT as a function of solvent polarity suggested that the through-space $\pi-\pi$ interactions between the aromatic rings in the C-shaped molecules stabilize their excited states. Collectively, the $\mathbf{B T}^{12}$ and $\mathbf{B B T}$ family of C-shaped molecules represent an interesting new class of fluorescent compounds whose emission colors span a wide range across the solvents studied, which suggests that they could be further explored as OLED materials ${ }^{45}$ or as solvatochromic polarity probes. ${ }^{6,35}$

\section{Experimental section}

\subsection{General}


Microwave-assisted reactions were carried out in a commercially available CEM Discover microwave unit, where the temperature in the reaction vessel was monitored in situ using an IR probe. The microwave power was set initially to $200 \mathrm{~W}$ and was automatically modulated by the microwave to reach and maintain the target reaction temperature. NMR spectroscopy experiments were conducted on either a $200 \mathrm{MHz}$ or a $400 \mathrm{MHz} \mathrm{NMR}$ spectrometer, as specified. Chemical shifts $(\delta)$ are reported in parts per million (ppm) and referenced to TMS $(\delta=0.00 \mathrm{ppm})$ for ${ }^{1} \mathrm{H}$ or residual solvent $(\delta=77.23 \mathrm{ppm})$ for ${ }^{13} \mathrm{C}$. For ${ }^{1} \mathrm{H}$ NMR spectroscopy, peak multiplicities are reported as s, singlet; $d$, doublet; $t$, triplet; $m$, multiplet; and dd, doublet of doublets. Melting points were determined in open capillaries with an electronic apparatus and are uncorrected. $\mathrm{Pd}\left(\mathrm{PPh}_{3}\right)_{4}$ was purchased from Strem Chemicals, Inc. All other solvents and reagents were purchased from commercial sources and used without any further purification. Column chromatography was performed using EMD silica gel 60 (230-400 mesh ASTM). TLC and preparative TLC were carried out using EMD TLC silica gel $60 \mathrm{~F}_{254}$ plates and on EMD $1 \mathrm{~mm}$ silica gel $60 \mathrm{~F}_{254}$ plates, respectively. High-resolution mass spectrometry was performed on a QTOF spectrometer. UV-vis absorption and fluorescence spectroscopy measurements were carried out in $1 \mathrm{~cm}$ path length quartz cuvettes at room

temperature. Relative quantum yields were measured using coumarin 153 in methanol as a reference. $^{39,44}$

\subsection{Experimental procedures}

4.2.1. 2,9-Diaza-5-bromotricyclo[8.4.0.0 $0^{3,8}$ ]tetradeca-1(10),3,5,7,9-pentaene (CQB). A $10 \mathrm{~mL}$ microwave vessel equipped with a stir bar was charged with $50 \mathrm{mg}(0.446 \mathrm{mmol})$ of 1,2 cyclohexanedione, $13 \mathrm{mg}(0.071 \mathrm{mmol})$ of zinc acetate, and $188 \mathrm{mg}(1.005 \mathrm{mmol})$ of 1,2- 
diamino-4-bromobenzene, followed by $2 \mathrm{~mL}$ of chlorobenzene. The headspace was purged with $\mathrm{N}_{2}$. The vessel was heated to $200{ }^{\circ} \mathrm{C}$ for 30 min with stirring in a CEM microwave. The resulting reaction mixture was filtered through a cotton plug, and the solvent was evaporated. The crude product was purified by column chromatography on silica gel (eluent: $20 \%$ ethyl acetate in hexanes), and the resulting pink solid was decolorized with activated carbon to give CQB as a white solid (90 mg; 77\% yield). CQB (mp 84.2-85.0 $\left.{ }^{\circ} \mathrm{C}\right):{ }^{1} \mathrm{H}$ NMR $\left(200 \mathrm{MHz}, \mathrm{CDCl}_{3}\right) \delta 8.14(\mathrm{~d}$, $J=2.0 \mathrm{~Hz}, 1 \mathrm{H}), 7.83(\mathrm{~d}, J=9 \mathrm{~Hz}, 1 \mathrm{H}), 7.39(\mathrm{dd}, J=8.9,2.1 \mathrm{~Hz}, 1 \mathrm{H}), 3.19-3.10(\mathrm{~m}, 4 \mathrm{H}), 2.08-$ $2.01(\mathrm{~m}, 4 \mathrm{H}) ;{ }^{13} \mathrm{C} \mathrm{NMR}\left(50 \mathrm{MHz}, \mathrm{CDCl}_{3}\right) \delta$ 155.4, 154.8, 142.1, 140.2, 132.6, 131.0, 130.0, 122.8, 33.4, 22.9. HRMS (TOF, ES+): $[\mathrm{M}+\mathrm{H}]^{+} \mathrm{m} / z$ calcd for $\mathrm{C}_{12} \mathrm{H}_{12} \mathrm{BrN}_{2}, 263.0184$; found, 263.0184 .

4.2.2. 2,9-Diaza-5-(2-thienyl)tricyclo[8.4.0.0 $\left.{ }^{3,8}\right]$ tetradeca-1(10),3,5,7,9-pentaene (CQT). A 10 $\mathrm{mL}$ microwave vessel was equipped with a stir bar, $35 \mathrm{mg}(0.133 \mathrm{mmol})$ of CQB, $51 \mathrm{mg}(0.399$ mmol) of 2-thienylboronic acid, $4 \mathrm{~mL}$ of toluene, and $1 \mathrm{~mL}$ of absolute ethanol. The reaction mixture was purged with $\mathrm{N}_{2}$ gas. A $29 \mathrm{mg}(0.025 \mathrm{mmol})$ portion of $\mathrm{Pd}\left(\mathrm{PPh}_{3}\right)_{4}$ was added followed by $0.46 \mathrm{~mL}$ of a $0.2 \mathrm{~g} / \mathrm{mL}$ solution of $\mathrm{Na}_{2} \mathrm{CO}_{3}$. The headspace was purged with $\mathrm{N}_{2}$ gas. The microwave vessel was heated to $120{ }^{\circ} \mathrm{C}$ for 30 min with stirring in a CEM microwave. The reaction mixture was transferred to a separatory funnel and extracted twice with ethyl acetate. The combined organic extracts were washed with water and then brine. The organic layer was dried over anhydrous $\mathrm{MgSO}_{4}$, and the solvent was removed in vacuo. The crude compound was purified by chromatography on silica gel with $30 \%$ ethyl acetate in hexanes as the eluent. CQT was isolated as an off-white crystalline solid (30 mg; 86\% yield). CQT (mp 103.9-106.7 ${ }^{\circ} \mathrm{C}$ w/decomp): ${ }^{1} \mathrm{H}$ NMR $\left(200 \mathrm{MHz}, \mathrm{CDCl}_{3}\right) \delta$ 8.21-8.19 (m, 1H), 7.96-7.95 (m, 2H), $7.50(\mathrm{dd}, J=$ 
3.6, $1.2 \mathrm{~Hz}, 1 \mathrm{H}), 7.37(\mathrm{dd}, J=5.1,1.1 \mathrm{~Hz}, 1 \mathrm{H}), 7.14(\mathrm{dd}, J=5.1,3.7 \mathrm{~Hz}, 1 \mathrm{H}), 3.20-3.14(\mathrm{~m}$, 4H), 2.09-2.02 (m, 4H); ${ }^{13} \mathrm{C} \mathrm{NMR}\left(50 \mathrm{MHz}, \mathrm{CDCl}_{3}\right) \delta$ 155.0, 154.1, 143.5, 141.7, 140.9, 135.1, 129.0, 128.6, 127.6, 126.3, 124.6, 124.2, 33.45, 33.39, 23.02, 23.00. HRMS (TOF, ES+): [M + $\mathrm{H}]^{+} \mathrm{m} / \mathrm{z}$ calcd for $\mathrm{C}_{16} \mathrm{H}_{15} \mathrm{~N}_{2} \mathrm{~S}, 267.0956$; found, 267.0966.

4.2.3. 2,9-Diaza-5-(5-(2'-thienyl)-2-thienyl)tricyclo[8.4.0.0 $\left.{ }^{3,8}\right]$ tetradeca-1(10),3,5,7,9-pentaene (CQBT). A $10 \mathrm{~mL}$ microwave vessel equipped with a stir bar was charged with $49 \mathrm{mg}(0.186$ $\mathrm{mmol}$ ) of CQB, $114 \mathrm{mg}$ (0.543 mmol) of 2,2'-bithiophene-5-boronic acid, $4 \mathrm{~mL}$ of toluene, and $1 \mathrm{~mL}$ of absolute ethanol. The reaction mixture was purged with $\mathrm{N}_{2}$ gas. A $0.645 \mathrm{~mL}$ portion of an aqueous $\mathrm{Na}_{2} \mathrm{CO}_{3}$ solution $(0.2 \mathrm{~g} / \mathrm{mL})$ was added, followed by $41 \mathrm{mg}(0.035 \mathrm{mmol})$ of $\operatorname{Pd}\left(\mathrm{PPh}_{3}\right)_{4}$. The headspace was purged with $\mathrm{N}_{2}$. The vessel was heated to $120{ }^{\circ} \mathrm{C}$ for 30 min with stirring in a CEM microwave. Upon completion, the contents were transferred to a separatory funnel, and the biphasic reaction mixture was extracted with ethyl acetate. The combined organic extracts were washed once with water and then with brine. The organic layer was dried over anhydrous $\mathrm{MgSO}_{4}$, and the solvent was removed in vacuo. The crude product was purified by column and preparative chromatography on silica gel (eluent: $30 \%$ ethyl acetate in hexanes) to give CQBT as a yellow solid (45 mg; 69\% yield). CQBT (mp 135.2-138.3 ${ }^{\circ} \mathrm{C}$ w/decomp): ${ }^{1} \mathrm{H}$ NMR $\left(400 \mathrm{MHz}, \mathrm{CDCl}_{3}\right) \delta 8.15(\mathrm{~d}, J=2.0 \mathrm{~Hz}, 1 \mathrm{H}), 7.94(\mathrm{~d}, J=8.8 \mathrm{~Hz}, 1 \mathrm{H}), 7.90(\mathrm{dd}, J=8.8$, $2.0 \mathrm{~Hz}, 1 \mathrm{H}), 7.39(\mathrm{~d}, J=3.6 \mathrm{~Hz}, 1 \mathrm{H}), 7.25-7.22(\mathrm{~m}, 2 \mathrm{H}), 7.18(\mathrm{~d}, J=4.0 \mathrm{~Hz}, 1 \mathrm{H}), 7.04(\mathrm{dd}, J=$ 4.8, 3.6 Hz, 1H), 3.16-3.15 (m, 4H), 2.06-2.02 (m, 4H); ${ }^{13} \mathrm{C}$ NMR (100 MHz, $\left.\mathrm{CDCl}_{3}\right) \delta 155.1$, $154.1,142.0,141.7,141.0,138.3,137.3,134.7,129.1,128.1,127.1,125.3,125.1,125.0,124.2$ 123.9, 33.5, 33.4, 23.00, 22.98. HRMS (TOF, ES+): $[\mathrm{M}+\mathrm{H}]^{+} \mathrm{m} / z$ calcd for $\mathrm{C}_{20} \mathrm{H}_{17} \mathrm{~N}_{2} \mathrm{~S}_{2}$, 349.0833; found, 349.0830 . 
4.2.4. (1 $(\alpha, 2 \beta, 4 \beta, 5 \alpha, 16 \alpha, 17 \beta, 19 \beta, 20 \alpha)-7,14,22,29$-Tetraaza-1,5,16,20-tetrachloro-31,31,32,32tetramethoxy-11,26-di(5-(2'-thienyl)-2-

thienyl)nonacyclo[18.10.1 $\left.11^{5,16} \cdot 0^{2,19} \cdot 0^{4,17} \cdot 0^{6,15} \cdot 0^{8,13} \cdot 0^{21,30} \cdot 0^{23,28}\right]$ dotriaconta6(15),7,9,11,13,21(30),22,24,26,28-decaene (anti-BBT). A $10 \mathrm{~mL}$ microwave vessel was equipped with a stir bar, $100 \mathrm{mg}(0.120 \mathrm{mmol})$ of anti-BBr, $101 \mathrm{mg}(0.481 \mathrm{mmol})$ of 2,2'bithiophene-5-boronic acid, $4 \mathrm{~mL}$ of toluene, and $1 \mathrm{~mL}$ of absolute ethanol. The reaction mixture was purged with $\mathrm{N}_{2}$ gas. A $26 \mathrm{mg}(0.022 \mathrm{mmol})$ portion of $\mathrm{Pd}\left(\mathrm{PPh}_{3}\right)_{4}$ was added, followed by $0.415 \mathrm{~mL}$ of a $0.2 \mathrm{~g} / \mathrm{mL}$ solution of $\mathrm{Na}_{2} \mathrm{CO}_{3}$. The headspace was purged with $\mathrm{N}_{2}$ gas. The microwave vessel was heated to $120{ }^{\circ} \mathrm{C}$ for 30 min with stirring in a CEM microwave. The solution was transferred to a separatory funnel and extracted twice with ethyl acetate. The combined organic extracts were washed once with water followed by another wash with brine. The organic layer was dried over anhydrous $\mathrm{MgSO}_{4}$. The solvent was removed in vacuo. The crude compound was purified by chromatography on silica gel two times with $30 \%$ ethyl acetate in hexanes as the eluent. Anti-BBT was isolated as a yellow solid (29 mg; $24 \%$ yield). Anti-BBT (mp $273{ }^{\circ} \mathrm{C}$ w/decomp): ${ }^{1} \mathrm{H}$ NMR $\left(400 \mathrm{MHz}, \mathrm{CDCl}_{3}\right) \delta 7.92(\mathrm{~d}, J=1.6 \mathrm{~Hz}, 2 \mathrm{H}), 7.70(\mathrm{~d}, J=8.8$ $\mathrm{Hz}, 2 \mathrm{H}), 7.46(\mathrm{dd}, J=8.6,2.2 \mathrm{~Hz}, 2 \mathrm{H}), 7.31(\mathrm{dd}, J=5.2,1.2 \mathrm{~Hz}, 2 \mathrm{H}), 7.27(\mathrm{dd}, J=3.4,1.0 \mathrm{~Hz}$ 2H), $7.24(\mathrm{~d}, J=4.0 \mathrm{~Hz}, 2 \mathrm{H}), 7.20(\mathrm{~d}, J=4.0 \mathrm{~Hz}, 2 \mathrm{H}), 7.09(\mathrm{dd}, J=5.2,3.6 \mathrm{~Hz}, 2 \mathrm{H}), 3.75(\mathrm{~s}$, $6 \mathrm{H}), 3.36(\mathrm{~s}, 6 \mathrm{H}), 2.91-2.88(\mathrm{~m}, 4 \mathrm{H}), 2.03-1.98(\mathrm{~m}, 2 \mathrm{H}),-1.05(\mathrm{~m}, 2 \mathrm{H}) ;{ }^{13} \mathrm{C} \mathrm{NMR}(100 \mathrm{MHz}$, $\left.\mathrm{CDCl}_{3}\right) \delta 152.7,151.7,141.9,141.2,140.9,139.0,137.3,135.3,129.2,128.3,127.3,125.7$, 125.2, 125.0, 124.4, 123.7, 111.4, 75.1, 75.0, 52.6, 52.2, 43.5, 43.3, 19.9. HRMS (TOF, ES+): $[\mathrm{M}+\mathrm{H}]^{+} \mathrm{m} / z$ calcd for $\mathrm{C}_{48} \mathrm{H}_{37} \mathrm{~N}_{4} \mathrm{O}_{4} \mathrm{~S}_{4} \mathrm{Cl}_{4}, 1001.0452$; found, 1001.0415 . 
4.2.5. $(1 \alpha, 2 \beta, 4 \beta, 5 \alpha, 16 \alpha, 17 \beta, 19 \beta, 20 \alpha)-7,14,22,29$-Tetraaza-1,5,16,20-tetrachloro-31,31,32,32tetramethoxy-10,26-di(5-(2'-thienyl)-2-

thienyl) $\left[18 \cdot 10 \cdot 1 \cdot 1^{5,16} \cdot 0^{2,19} \cdot 0^{4,17} \cdot 0^{6,15} \cdot 0^{8,13} 0^{21,30} \cdot 0^{23,28}\right]$ dotriaconta-

6(15),7,9,11,13,21(30),22,24,26,28-decaene (syn-BBT). A $10 \mathrm{~mL}$ microwave vessel equipped with a stir bar was charged with $89 \mathrm{mg}(0.107 \mathrm{mmol})$ of syn-BBr, $135 \mathrm{mg}(0.643 \mathrm{mmol})$ of 2,2'bithiophene-5-boronic acid, $4 \mathrm{~mL}$ of toluene, and $1 \mathrm{~mL}$ of absolute ethanol. The reaction mixture was purged with $\mathrm{N}_{2}$ gas. A $0.37 \mathrm{~mL}$ portion of an aqueous $\mathrm{Na}_{2} \mathrm{CO}_{3}$ solution $(0.2 \mathrm{~g} / \mathrm{mL})$ was added, followed by $23 \mathrm{mg}(0.020 \mathrm{mmol})$ of $\mathrm{Pd}\left(\mathrm{PPh}_{3}\right)_{4}$. The headspace was purged with $\mathrm{N}_{2}$. The vessel was heated to $120^{\circ} \mathrm{C}$ for 30 min with stirring in a CEM microwave. Upon completion, the contents were transferred to a separatory funnel, and the biphasic reaction mixture was extracted with ethyl acetate. The combined organic extracts were washed once with water and once with brine. The organic layer was dried over anhydrous $\mathrm{MgSO}_{4}$, and the solvent was removed in vacuo. The crude product was purified by column chromatography on silica gel with $30 \%$ ethyl acetate in hexanes as the eluent. Syn-BBT was isolated as an orange/yellow solid (20 mg; 19\% yield). Syn-BBT (mp 301-304.5 ${ }^{\circ} \mathrm{C}$ w/decomp): ${ }^{1} \mathrm{H}$ NMR (200 MHz, $\left.\mathrm{CDCl}_{3}\right) \delta 7.93$ (m, 2H), 7.74-7.68 (m, 4H), $7.19(\mathrm{dd}, J=5.2,1.2 \mathrm{~Hz}, 2 \mathrm{H}), 7.07(\mathrm{~d}, J=4.0 \mathrm{~Hz}, 2 \mathrm{H}), 7.05(\mathrm{dd}, J=3.6,1.2$ $\mathrm{Hz}, 2 \mathrm{H}), 6.96(\mathrm{dd}, J=5.2,3.6 \mathrm{~Hz}, 2 \mathrm{H}), 6.81(\mathrm{~d}, J=3.6 \mathrm{~Hz}, 2 \mathrm{H}), 3.74(\mathrm{~s}, 6 \mathrm{H}), 3.35(\mathrm{~s}, 6 \mathrm{H}), 2.93-$ $2.88(\mathrm{~m}, 4 \mathrm{H}), 2.05-1.98(\mathrm{~m}, 2 \mathrm{H}),-1.00(\mathrm{~m}, 2 \mathrm{H}) ;{ }^{13} \mathrm{C} \mathrm{NMR}\left(100 \mathrm{MHz}, \mathrm{CDCl}_{3}\right) \delta 152.8,151.5$, $141.9,140.9,140.6,138.5,137.4,135.5,129.3,128.1,127.2,125.5,124.9,124.8,124.0,123.5$, 111.4, 75.10, 75.06, 52.6, 52.2, 43.6, 43.3, 19.92, 19.87. HRMS (TOF, ES+): $[\mathrm{M}+\mathrm{H}]^{+} \mathrm{m} / z$ calcd for $\mathrm{C}_{48} \mathrm{H}_{37} \mathrm{~N}_{4} \mathrm{O}_{4} \mathrm{~S}_{4} \mathrm{Cl}_{4}, 1001.0452$; found, 1001.0433 .

\section{Acknowledgements}


The authors would like to thank Dr. Karen Walker for her assistance in carrying out the $400 \mathrm{MHz}$ NMR spectroscopy experiments at Vassar College. NMR instrumentation was provided by the US National Science Foundation (NSF-MRI Grant No. 1526982, J. Tanski P.I., T. Garrett co-P.I.). We also thank Laura Wickham for her assistance with compound purification and the Marist College School of Science for financial support.

\section{Supplementary data}

Supplementary data associated with this article $\left({ }^{1} \mathrm{H}\right.$ and ${ }^{13} \mathrm{C}$ NMR spectra) can be found in the online version, at:

\section{References and notes}

1. Roncali, J. Macromol. Rapid Commun. 2007, 28, 1761-1775.

2. Grimsdale, A. C.; Chan, K. L.; Martin, R. E.; Jokisz, P. G.; Holmes, A. B. Chem. Rev. 2009, 109, 897-1091.

3. Cornil, J.; Beljonne, D.; Calbert, J.-P.; Brédas, J.-L. Adv. Mater. 2001, 13, 1053-1067.

4. Murphy, A. R.; Fréchet, J. M. J. Chem. Rev. 2007, 107, 1066-1096.

5. Spitler, E. L.; Monson, J. M.; Haley, M. M. J. Org. Chem. 2008, 73, 2211-2223.

6. Gers, C. F.; Nordmann, J.; Kumru, C.; Frank, W.; Müller, T. J. J. Org. Chem. 2014, 79, $3296-3310$.

7. Yao, L.; Sun, S.; Xue, S.; Zhang, S.; Wu, X.; Zhang, H.; Pan, Y.; Gu, C.; Li, F.; Ma, Y. J. Phys. Chem. C 2013, 117, 14189-14196.

8. Apperloo, J. J.; Groenendaal, L. B.; Verheyen, H.; Jayakannan, M.; Janssen, R. A. J.; Dkhissi, A.; Beljonne, D.; Lazzaroni, R.; Brédas, J.-L. Chem. Eur. J. 2002, 8, 2384-2396. 
9. An, Z.; Odom, S. A.; Kelley, R. F.; Huang, C.; Zhang, X.; Barlow, S.; Padilha, L. A.; Fu, J.; Webster, S.; Hagan, D. J.; Van Stryland, E. W.; Wasielewski, M. R.; Marder S. R. J. Phys. Chem. A 2009, 113, 5585-5593.

10. Khanasa, T.; Prachumrak, N.; Rattanawan, R.; Jungsuttiwong, S.; Keawin, T.; Sudyoadsuk, T.; Tuntulani, T.; Promarak, V. J. Org. Chem. 2013, 78, 6702-6713.

11. Chou, T.-C.; Hwa, C.-L.; Lin, J.-J.; Liao, K.-C.; Tseng, J.-C. J. Org. Chem. 2005, 70, 9717-9726.

12. DeBlase, C. R.; Finke, R. T.; Porras, J. A.; Tanski, J. M.; Nadeau, J. M. J. Org. Chem. 2014, $79,4312-4321$.

13. Chou, T.-C.; Liao, K.-C.; Lin, J.-J. Org. Lett. 2005, 7, 4843-4846.

14. Thirion, D.; Poriel, C.; Barrière, F.; Métivier, R.; Jeannin, O.; Rault-Berthelot, J. Org. Lett. 2009, 11, 4794-4797.

15. Thirion, D.; Poriel, C.; Métivier, R.; Rault-Berthelot, J.; Barrière, F.; Jeannin, O. Chem. Eur. J. 2011, 17, 10272-10287.

16. Leung, M.-k.; Viswanath, M. B.; Chou, P.-T.; Pu, S.-C.; Lin, H.-C.; Jin, B.-Y. J. Org. Chem. 2005, 70, 3560-3568.

17. Pognon, G.; Boudon, C.; Schenk, K. J.; Bonin, M.; Bach, B.; Weiss, J. J. Am. Chem. Soc. 2006, 128, 3488-3489.

18. Alamiry, M. A. H.; Benniston, A. C.; Copley, G.; Harriman, A.; Howgego, D. J. Phys. Chem. A 2011, 115, 12111-12119.

19. Giaimo, J. M.; Lockard, J. V.; Sinks, L. E.; Scott, A. M.; Wilson, T. M.; Wasielewski, M. R. J. Phys. Chem. A 2008, 112, 2322-2330.

20. Knoblock, K. M.; Silvestri, C. J.; Collard, D. M. J. Am. Chem. Soc. 2006, 128, 13680-13681. 
21. Brédas, J.-L.; Beljonne, D.; Coropceanu, V.; Cornil, J. Chem. Rev. 2004, 104, 4971-5003.

22. Riley, K. E.; Hobza, P. Acc. Chem. Res. 2013, 46, 927-936.

23. Schneebeli, S. T.; Kamenetska, M.; Cheng, Z.; Skouta, R.; Friesner, R. A.; Venkataraman, L.; Breslow, R. J. Am. Chem. Soc. 2011, 133, 2136-2139.

24. Jagtap, S. P.; Mukhopadhyay, S.; Coropceanu, V.; Brizius, G. L.; Brédas, J.-L.; Collard, D. M. J. Am. Chem. Soc. 2012, 134, 7176-7185.

25. Hartnett, P. E.; Margulies, E. A.; Matte, H. S. S. R.; Hersam, M. C.; Marks, T. J.; Wasielewski, M. R. Chem. Mater. 2016, 28, 3928-3936.

26. Thirion, D.; Romain, M.; Rault-Berthelot, J.; Poriel, C. J. Mater. Chem. 2012, 22, $7149-7157$.

27. Yoo, H.; Yang, J.; Yousef, A.; Wasielewski, M. R.; Kim, D. J. Am. Chem. Soc. 2010, 132, 3939-3944.

28. Jagtap, S. P.; Collard, D. M. J. Am. Chem. Soc. 2010, 132, 12208-12209.

29. Ohkita, H.; Ito, S.; Yamamoto, M.; Tohda, Y.; Tani, K. J. Phys. Chem. A 2002, 106, $2140-2145$.

30. Benten, H.; Ohkita, H.; Ito, S.; Yamamoto, M.; Sakumoto, N.; Hori, K.; Tohda, Y.; Tani, K.; Nakamura, Y.; Nishimura, J. J. Phys. Chem. B 2005, 109, 19681-19687.

31. Bartholomew, G. P.; Bazan, G. C. Acc. Chem. Res. 2001, 34, 30-39.

32. Morisaki, Y.; Sawamura, T.; Murakami, T.; Chujo, Y. Org. Lett. 2010, 12, 3188-3191.

33. Rathore, R.; Abdelwahed, S. H.; Guzei, I. A. J. Am. Chem. Soc. 2003, 125, 8712-8713.

34. Garnier, F. Acc. Chem. Res. 1999, 32, 209-215.

35. Reichardt, C. Chem. Rev. 1994, 94, 2319-2358.

36. Calculated using Spartan '16 for Windows (Version 1.1.0) by Wavefunction, Inc. 
37. Reichardt, C.; Welton, T. Solvents and Solvent Effects in Organic Chemistry, $4^{\text {th }}$ Ed.; WileyVCH: Weinheim, 2011.

38. Lee, S. A.; Hotta, S.; Nakanishi, F. J. Phys. Chem. A 2000, 104, 1827-1833.

39. Boens, N.; Qin, W.; Basarić, N.; Hofkens, J.; Ameloot, M.; Pouget, J.; Lefèvre, J.-P.; Valeur, B.; Gratton, E.; vandeVen, M.; Silva, N. D., Jr.; Engelborghs, Y.; Willaert, K.; Sillen, A.; Rumbles, G.; Phillips, D.; Visser, A. J. W. G.; van Hoek, A.; Lakowicz, J. R.; Malak, H.; Gryczynski, I.; Szabo, A. G.; Krajcarski, D. T.; Tamai, N.; Miura, A. Anal. Chem. 2007, 79, 2137-2149.

40. The analysis was limited to solvents in which only a single, broad emission band was observed.

41. Yamamoto, T.; Zhou, Z.-h.; Kanbara, T.; Shimura, M.; Kizu, K.; Maruyama, T.; Nakamura, Y.; Fukuda, T.; Lee, B.-L.; Ooba, N.; Tomaru, S.; Kurihara, T.; Kaino, T.; Kubota, K.; Sasaki, S. J. Am. Chem. Soc. 1996, 118, 10389-10399.

42. Choi, Y.-S.; Lee, W.-h.; Kim, J.-R.; Lee, S.-K.; Shin, W.-S.; Moon, S.-J.; Park, J.-W.; Kang, I.-N. Bull. Korean Chem. Soc. 2011, 32, 417-423.

43. Lakowicz, J. R. Principles of Fluorescence Spectroscopy, $3^{\text {rd }}$ Ed.; Springer: New York, 2006.

44. Fery-Forgues, S.; Lavabre, D. J. Chem. Educ. 1999, 76, 1260-1264.

45. Zhu, X.-H.; Peng, J.; Cao, Y.; Roncali, J. Chem. Soc. Rev. 2011, 40, 3509-3524. 


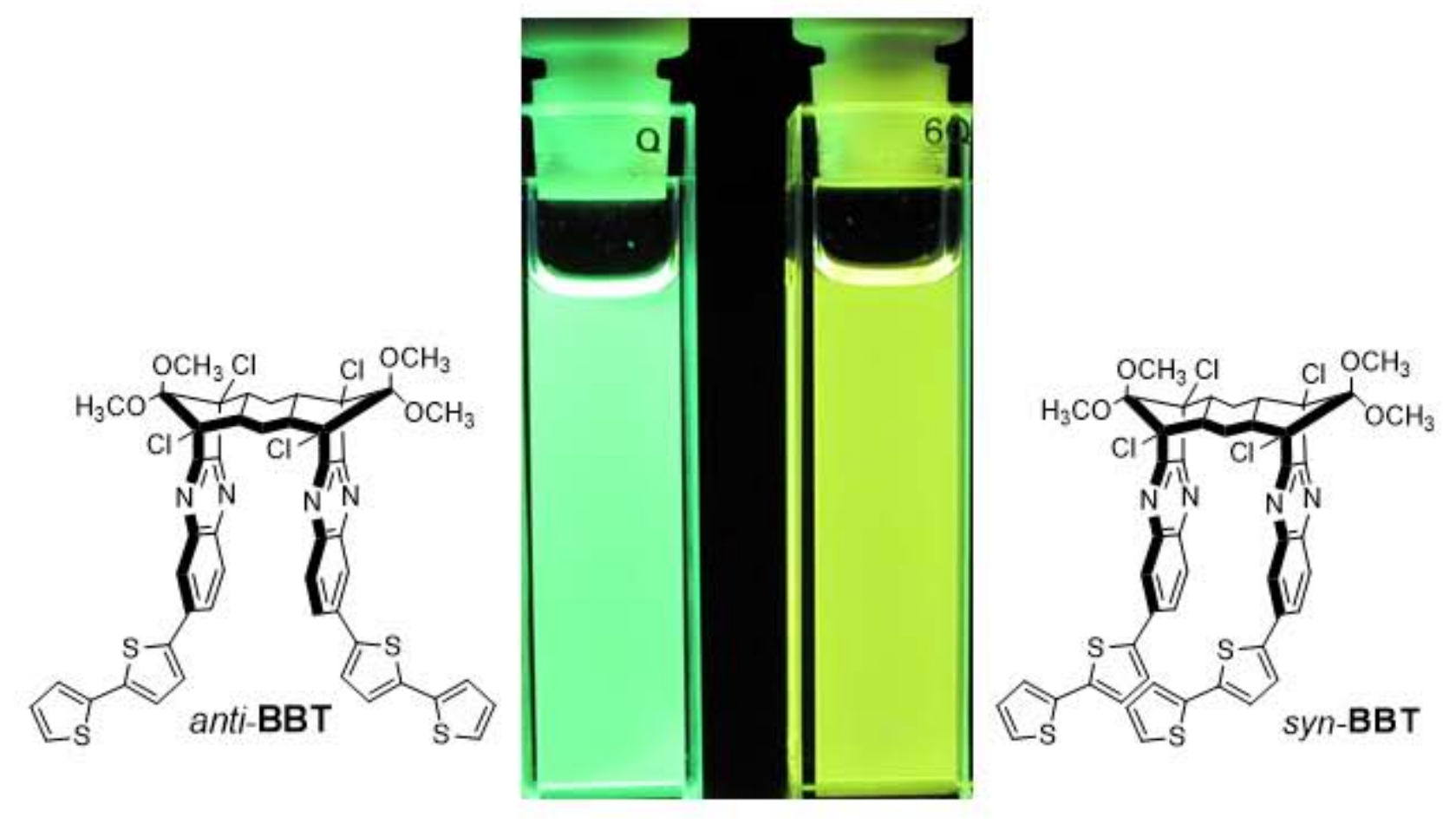

